becomes visible as a millisecond pulsar. Accretion of matter onto the deep gravitational potential well of the neutron star results in the copious production of $\mathrm{X}$ rays, so the mass transfer stage is usually identified with the luminous low-mass X-ray binaries (LMXBs), of which there are about a hundred in the Galaxy.

Several problems remain with this physically plausible model, however. First is the well known 'birthrate problem'. There does not seem to be an adequate supply of LMXBs to serve as progenitors for the very abundant millisecond pulsars $^{5}$. Second, although LMXBs are thought to contain neutron stars spinning at millisecond periods, considerable effort has uncovered no evidence for millisecond pulsations from any of them ${ }^{6}$. Indeed, there is indirect evidence suggesting that at least some LMXBs may be harbouring slowly rotating neutron stars ${ }^{7}$. Third, the origin and evolution of magnetic fields of neutron stars is not understood within the model. If millisecond pulsars are simply ordinary pulsars that have been spun up, they have somehow dispensed with 99.99 per cent, but no more, of their original magnetic fields. Finally, although the uncertainties are still high, the recent mass determination of pulsar $1855+09$ (ref. 8) suggests that it may be a 'light' neutron star, with mass $\sim 1.25 M_{\odot}$. This result, if confirmed, may prove difficult to reconcile with the substantial mass transfer required to spin the pulsar up to millisecond periods.

\section{Achilles' heel}

In the context of the standard picture, several models for the origin of magnetic fields in millisecond pulsars have been suggested. Each seems to have its own Achilles' heel, as well as poorly understood physical underpinnings. One such model $^{9}$ relates accretion history to field reduction. Low magnetic fields are then a consequence of the large mass accreted in LMXBs relative to massive binary systems or isolated pulsars.

Another model ${ }^{10}$ argues that the field reduction is related to the slowing down of the neutron star. In this model, there is a phase in the early stages of spin-up when the neutron star is spun down by accretion before getting spun up. During the spin down phase, most of the original field is transferred from the interior to the surface where it can decay. Thus the asymptotic field strength is determined by the longest period during spin down. But difficulties arise in explaining the high magnetic fields observed for some very slowly rotating neutron stars in high-mass binary systems.

Neither model can satisfactorily explain the field gap. In the absence of a physical reason to limit field reduction, both models predict a potentially large class of rapidly rotating neutron stars with very low magnetic field strengths $\left(\ll 10^{8} \mathrm{G}\right)$ thereby accentuating the birthrate problem.

The shortcomings in the standard model are sufficiently troubling that alternative models predicting direct formation of millisecond pulsars (that is, without spin-up by accretion) must be considered. One such model proposes formation by the accretion-induced collapse of massive white dwarfs. The birthrate problem disappears, as LMXBs are no longer relevant and the field gap is explained by assumption. Accretioninduced collapse has its own difficulties, however, starting with a fundamental uncertainty over whether white dwarfs can accrete matter without catastrophic destruction of the star in a type Ia supernova. The kinematics of millisecond pulsars as inferred by direct measurements of velocities and the observed isotropy of their local population suggests that velocity kicks are needed at birth. The proper motion of J0437's white dwarf companion has already been measured ${ }^{4}$ and the inferred velocity is reasonably high, about $80 \mathrm{~km} \mathrm{~s}^{-1}$. It remains to be seen whether accretion-induced collapse can satisfactorily explain the kinematics of such pulsars.

The rapid discovery rate of millisecond pulsars offers the hope of resolving their origin. Precise measurements of the masses in systems such as PSR1855+08 offer the most direct clue to the accretion history. The current observational selection effects that limit the sensitivity of searches for very fast (and potentially very low magnetic field) pulsars must be beaten down; if the tight clustering of magnetic field strengths holds up, this is telling us something fundamental about the origin of magnetic fields in neutron stars. Perhaps most of all we need more velocity measurements, made by timing, verylong-baseline interferometry or optical spectroscopy of bow shocks (if present, as in J0437) if we are to distinguish between the evolutionary models.

S. R. Kulkarni and S. E. Thorsett are in the Owens Valley Radio Observatory, California Institute of Technology, Pasadena, California 91125, USA.

1. Bell, J. F., Bailes, M. \& Bessell, M. S. Nature 364, 603-605 (1993).

2. Johnston, S et al. Nature 361, 613-615 (1993).

3. Bailyn, C. D. Astrophys. J. 411, L83-L85 (1993).

4. Danziger, I. J., Baade. D. \& Della Valle M. Astr. Astrophys. (in the press)

5. Bailes, M. \& Johnston, S. Mon. Not. R. astr. Soc. 252. 277-281 (1991).

6. Hertz, P., Norris, J. P., Wood. K. S., Vaughan, B. A. \& Michelson, P. F. Astrophys. J. 354, 267-272 (1990)

7. Kulkarni, S. R., Navarro, J., Vasisht, G., Tanaka, Y. \& Fumiaki, N. in X-Ray Binaries and Recycled Pulsars (eds van den Heuvel, E. P. J. \& Rappaport, S. A.) 99-105 (NATO ASI Series, Kluwer, Dordrecht)

8. Ryba, M. F. \& Taylor, J. H. Astrophys. J. 371, 739-748 (1991).

9. Shibazaki, N., Murakami, T., Shaham. J.\& Nomoto, K. Nature 342, 656-658 (1989).

10. Srinivasan, G., Bhattacharya, D., Muslimov, A. G. \& Tsygan, A. I. Curr. Sci. 59, 31-38 (1990).

\section{Currents of water}

MANY domestic technologies, such as washing, drying, cleaning, dehumidifying and so on, are merely complicated ways of moving small amounts of water.

Daedalus wants to simplify them.

He points out that many ions, such as those of sodium and hydrogen, dissolve in water to a hydrated form: $\mathrm{Na}\left(4 \mathrm{H}_{2} \mathrm{O}\right)^{+}$, $\mathrm{H}_{3} \mathrm{O}^{+}$and so on. Drive them through the solution by an applied voltage, and the water comes too. But when these ions move through a solid lattice (as do hydrogen ions in the glass electrode of a $\mathrm{pH}$ meter, sodium ions in the beta. alumina of a sodium-sulphur battery, and many positive ions in certain ionexchange resins), they lose this water. So says Daedalus, imagine a long semipermeable tube containing many alternate sections of aqueous gel and solid electrolyte. Put a voltage across the ends of the tube, and all the ions will move. Each time an ion traverses a liquidto-solid junction, it will be stripped of its water molecules; each time it passes a solid-to-liquid one, it will pick up new water. Now fold the conductor into a zig. zag such that one set of junctions forms one side of flat sheet, while the converse set forms the other. Switch on the current, and the sheet will absorb water on one face, and exude it from the other. Daedalus has invented an elegant electric water-pump with no moving parts.

DREADCO engineers are now realizing this vision. Their goal is a sort of electric flannel, each of whose innumerable fibre loops is a tiny water-pump element. It will soak up water avidly on one side, and discharge it on the other. It should be very efficient. The voltage needed to drive the ions into one set of junctions will be provided by their emergence from the other set. A little water will be electrolysed at the two end-electrodes, but at a trivial energetic cost.

DREADCO's electric dishcloths and towels will have an avid and untiring thirst for water. Sufficient electric heating will be designed into them to discharge it as vapour. By contrast, DREADCO's electric mop will suck up dirty water and discharge clean water ahead of it. One mop-load of water will thus serve to clean the whole floor. A simple reversal of the current will then float the accumulated dirt off the active surface. Daedalus even plans a batteryoperated electric raincoat, sweat-shirt and socks, to keep the wearer dry and comfortable in extremes of rain or sweaty exertion. He is also devising an electric damp-proof course for houses. Silently and unobtrusively, it will pump water out of the house. It will combine air-conditioning, dehumidification and rot-proofing. David Jones 